\title{
Effect of prepolymer architecture on the network structure formed by AB-type crosslink-coupling
}

\author{
Shinji Kondo, Ung-il Chung and Takamasa Sakai \\ An ideal polymer network free from any heterogeneity has been sought for decades to understand the molecular model for \\ rubber elasticity. Recently, we developed a new crosslinking method, 'crosslink-coupling.' Unlike the conventional 'end- \\ crosslinking' method, the crosslink-coupling method yielded an extremely homogeneous network; this result suggests that \\ the prepolymer architecture is vital for the suppression of heterogeneity. The differences in prepolymer architecture between \\ end-linking and crosslink-coupling are the 'sizes' and 'functionalities' of the prepolymers. In this study, we focus on the \\ prepolymer size and investigate the mechanical properties of the polymer network formed from mutually reactive tetra-arm \\ polyethylene glycol prepolymers with different sizes (size-mismatched Tetra-PEG gels). Our experiments revealed that the elastic \\ modulus, the fracture energy and the ultimate elongation ratio of the size-mismatched Tetra-PEG gels had tendencies similar \\ to those of conventional Tetra-PEG gels. From these results, we conclude that under these conditions, the size-mismatched \\ Tetra-PEG gels have the same degree of homogeneity as the conventional Tetra-PEG gels, and the difference in prepolymer size \\ does not affect the homogeneity.
}

Polymer Journal (2014) 46, 14-20; doi:10.1038/pj.2013.65; published online 31 July 2013

Keywords: heterogeneities; hydrogels; polymer network structure; prepolymers

\section{INTRODUCTION}

Polymer gels are three-dimensional polymer networks crosslinked by physical or chemical interactions and swollen in solvent. One popular method for the formation of polymer gels is the radical polymerization of monomers and crosslinkers. ${ }^{1,2}$ Because monomers and crosslinkers react at random, the resultant network has a substantial number of heterogeneities, ${ }^{3,4}$ including spatial (heterogeneous distribution of crosslinks), connectivity (dangling chains and elastically ineffective loops) and topological (trapped entanglements) heterogeneities. ${ }^{5,6}$ Such heterogeneities are inevitable in conventional polymer gels and prohibit the precise control and prediction of the physical properties of the gels; this lack of control and predictability severely limits the scope of the applications of hydrogels. Thus, the establishment of principles for the construction of ideally homogeneous polymer networks is a significant challenge not only for understanding molecular models for the physical properties of the networks, but also for achieving flexibility in the design of polymer gels.

A popular approach toward the achievement of the ideal network is the 'end-crosslinking' method, which forms a polymer network from AB-type polycondensation of telechelic prepolymers and multifunctional crosslinkers (Figure 1a). ${ }^{7}$ However, the resultant network contains a substantial amount of heterogeneity. ${ }^{8,9}$ Thus, 'end-crosslinking' is not an appropriate method for the construction of ideal polymer networks.
Recently, we developed Tetra-PEG gels through our new networkformation method, 'crosslink-coupling;' the network is formed by the combination of two mutually reactive tetra-arm prepolymers with the same shapes (Figure 1c). ${ }^{10-12}$ Our previous study revealed that Tetra-PEG gels form an extremely homogeneous polymer network with a small number of structural defects. ${ }^{13-15}$ Although the connectivity and spatial heterogeneities were observed, the degree of heterogeneity is much smaller than that for conventional gels. ${ }^{16-19}$

What is the key to suppressing the heterogeneity? As shown in Figure 1a and $c$, both the end-crosslinking and crosslink-coupling methods, respectively, can lead to an ideal network structure if the reaction proceeds ideally. However, end-crosslinking yields a heterogeneous network, whereas crosslink-coupling yields a homogeneous network. These experimental findings indicate that the prepolymer architecture is vital in suppressing the heterogeneity. The differences in the prepolymer architecture for the end-linking and crosslinkcoupling are the 'size' and 'functionality' of the prepolymers; end-linking uses prepolymers with 'different sizes' and 'different functionalities,' whereas the crosslink-coupling uses prepolymers with the 'same sizes' and 'same functionalities'.

In this study, we try to investigate the effect of 'size.' We form polymer networks from the AB-type crosslink-coupling of prepolymers with 'different sizes' but the 'same functionalities;' we call these networks size-mismatched Tetra-PEG gels (Figure 1b). We investigate the reaction efficiency $(p)$ of these networks using infrared 
measurements, the elastic modulus $(G)$ and the ultimate elongation ratio $\left(\lambda_{\max }\right)$ using stretching measurements and the fracture energy $\left(T_{0}\right)$ using tearing measurements. By comparing these mechanical properties with those of conventional Tetra-PEG gels and with the predictions from models, we investigate the effect of the prepolymer architecture on the network structure formed by AB-type crosslinkcoupling.

\section{EXPERIMENTAL PROCEDURE}

\section{Characterization of Tetra-PEG modules}

Tetra-amine-terminated PEG (Tetra-PEG- $\mathrm{NH}_{2}$ ) and tetra-NHS-terminated PEG (Tetra-PEG-OSu) were purchased from NICHIYU (Tokyo, Japan). Here, NHS is $N$-hydroxysuccinimide. The details of the preparation of Tetra-PEG$\mathrm{NH}_{2}$ and Tetra-PEG-OSu were reported previously. ${ }^{10}$ The molecular weight, the polydispersity, the end-group functionality $\left(X_{0}\right)$, and the overlapping polymer volume fraction $\left(\phi^{*}\right)$ of the Tetra-PEG gels are shown in Supplementray Table 1 in the Supplementray Information.

\section{Fabrication of Tetra-PEG gels}

We combined three different molecular weights $\left(M_{\mathrm{w}}\right)$ of TetraPEG-NH ${ }_{2}\left(M_{\mathrm{w}}=5 \mathrm{k}, 10 \mathrm{k}\right.$ and $\left.20 \mathrm{k}\right)$ and Tetra-PEG-OSu $\left(M_{\mathrm{w}}=5 \mathrm{k}, 10 \mathrm{k}\right.$ and $20 \mathrm{k}$ ) to fabricate Tetra-PEG gels composed of size-mismatched TetraPEG-NH $\mathrm{NH}_{2}$ and Tetra-PEG-OSu (Table 1). Equimolar amounts of Tetra-PEG$\mathrm{NH}_{2}$ and Tetra-PEG-OSu were dissolved in phosphate buffer ( $\mathrm{pH}$ 7.4) and phosphate-citric acid buffer ( $\mathrm{pH}$ 5.8), respectively. The corresponding initial polymer volume fractions, $\phi_{0}$, were between 0.050 and 0.096 (mass density $=1.129 \mathrm{~g} \mathrm{~cm}^{-3}$ ). The ionic strengths of the buffers were chosen to

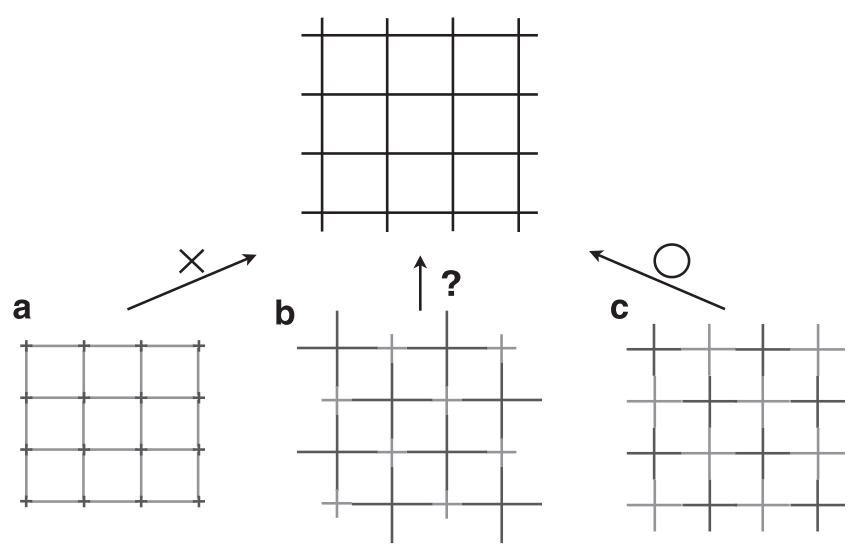

Figure 1 Polymer network structure formed from AB-type polycondensation of prepolymers with different architectures: (a) end-linking reaction, (b) size-mismatched crosslink-coupling and (c) conventional crosslinkcoupling. A full color version of this figure is available at Polymer Journal online.

Table 1 The combinations of prepolymers, the overlapping polymer volume fraction $\left(\phi^{*}\right)$ and the ionic strength of the buffers for each initial volume fraction $\left(\phi_{0}\right)$ in the Tetra-PEG gels

\begin{tabular}{llcccc}
\hline & & \multicolumn{5}{c}{ lonic strength of the buffers (mM) } \\
\cline { 3 - 6 } $\begin{array}{l}\text { Combinations } \\
\text { of prepolymers }\end{array}$ & $\phi^{*}$ & $\phi_{0}=0.050$ & $\phi_{0}=0.066$ & $\phi_{0}=0.081$ & $\phi_{0}=0.096$ \\
\hline $5 \mathrm{k}$ vs 10k & 0.080 & 50 & 50 & 100 & 100 \\
$5 \mathrm{k}$ vs 20k & 0.066 & 25 & 50 & 50 & 100 \\
$10 \mathrm{k}$ vs 20k & 0.048 & 25 & 25 & 50 & 50 \\
\hline
\end{tabular}

control the reaction rate (Table 1). ${ }^{20,21}$ The two solutions were mixed, and the resulting solution was poured into the mold. We waited for at least $12 \mathrm{~h}$ for the completion of the reaction before the subsequent experiment was performed.

\section{Stretching measurements}

The stretching measurements were performed on a dumbbell shape $(2 \mathrm{~mm}$ thick) at room temperature with a mechanical testing apparatus (Autograph AG-X plus; SHIMADZU, Kyoto, Japan) at a crosshead speed of $60 \mathrm{~mm} \mathrm{~min}^{-1}$, which corresponds to a strain rate at the gauge section of $\sim 2$ per min. During the stretching, the distance between the gauge points was measured with a CCD camera (XCL-5005CR; SONY, Tokyo, Japan). Because the gel samples were used in the as-prepared state, the stretching was performed in air. The stress-strain relations obtained here corresponded to the equilibrium relations without time effects because no appreciable relaxation was observed for the stress after the imposition of a constant large strain at this crosshead speed. We stretched a sample repeatedly and confirmed that the evaporation of water did not affect the experimental results. At least four samples were tested for each network concentration, and the observed moduli were arithmetically averaged.

\section{Infrared measurements}

After the stretching measurements, the gel samples were soaked in $\mathrm{H}_{2} \mathrm{O}$ for 2 days at room temperature and then were dried. The dry samples were cut into thin films (thickness: $40 \mu \mathrm{m}$ ) with a Microtome (SM2000R, Leica, Wetzlar, Germany). These samples were swollen with $\mathrm{D}_{2} \mathrm{O}$ until equilibrium was reached. The infrared spectra of these samples were obtained at $25^{\circ} \mathrm{C}$ with a JASCO FT-IR-6300 equipped with a deuterated triglycine sulfate (DTGS) detector, in which 128 scans were summed at a resolution of $4 \mathrm{~cm}^{-1}$ for each sample. At least two samples were tested for each network concentration.

\section{Tearing measurements}

The tearing measurements were performed at room temperature with a mechanical testing apparatus (Autograph AG-X plus; SHIMADZU, Kyoto, Japan). Because the gel samples were used in the as-prepared state, the tearing was performed in air. The gels were cut into the shape specified by JIS K 6252 at $1 / 2$ size $(50 \mathrm{~mm} \times 7.5 \mathrm{~mm} \times 1 \mathrm{~mm}$, with an initial notch of $20 \mathrm{~mm})$. The two arms of the test samples were clamped, and one arm was pulled downward at a constant velocity of $40 \mathrm{~mm} \mathrm{~min}^{-1}$, whereas the other arm remained stationary. The tearing force $F$ was recorded.

\section{RESULTS AND DISCUSSION}

\section{Reaction efficiency}

Conventional Tetra-PEG gels are fabricated from Tetra-PEG-NH $\mathrm{N}_{2}$ and Tetra-PEG-OSu with equal molecular weights. ${ }^{10}$ To investigate the effect of the size mismatch of the prepolymers, we fabricated $5-10 \mathrm{k}$, $5-20 \mathrm{k}$ and $10-20 \mathrm{k}$ Tetra-PEG gels, where $5-10 \mathrm{k}$ indicates that equimolar Tetra-PEG-NH $\mathrm{NH}_{2} \quad\left(5 \mathrm{~kg} \mathrm{~mol}^{-1}\right)$ and Tetra-PEG-OSu $\left(10 \mathrm{~kg} \mathrm{~mol}^{-1}\right)$ were mixed (Table 1$)$.

First, we measured the $p$ of the $5-10 \mathrm{k}, 5-20 \mathrm{k}$ and $10-20 \mathrm{k}$ TetraPEG gels using infrared measurements (Figure 2). $p$ was almost constant as a function of $\phi_{0}$ and was $0.88-0.93$, which is as high as that of conventional Tetra-PEG gels $(0.82-0.95)$. These results suggest that the $p$ only depends on the reaction group and not on the polymer size.

\section{Elastic modulus}

We performed stretching tests for the $5-10 \mathrm{k}, 5-20 \mathrm{k}$ and $10-20 \mathrm{k}$ Tetra-PEG gels. According to the linear elasticity theory, ${ }^{22}$ we estimated the elastic moduli $(G)$ from the initial slopes of the stress $(\sigma)$-elongation $(\lambda)$ curves. The elastic moduli are predicted from the affine network model $\left(G_{\mathrm{af}}\right)$ and from the phantom network model $\left(G_{\mathrm{ph}}\right)$ to be as follows: 


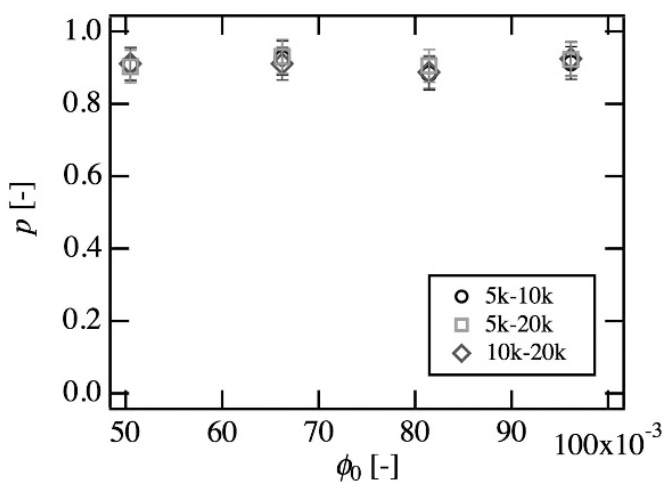

Figure 2 The reaction efficiency $(p)$ as a function of the initial polymer fraction $\left(\phi_{0}\right)$ in the Tetra-PEG gels $(5 \mathrm{k}-10 \mathrm{k}$, circles; $5 \mathrm{k}-20 \mathrm{k}$, squares; $10 \mathrm{k}-20 \mathrm{k}$, rhombuses). A full color version of this figure is available at Polymer Journal online.

$$
G_{\mathrm{af}}=v \cdot k_{\mathrm{B}} T \quad(\text { affine network model })
$$

$$
G_{\mathrm{ph}}=(v-\mu) \cdot k_{\mathrm{B}} T \quad(\text { phantom network model })
$$

where $k_{\mathrm{B}}$ is Boltzmann's constant and $T$ is the absolute temperature. The number densities of the active crosslinks $(\mu)$ and the elastically effective chains $(v)$ for the tetra-functional network are predicted based on a tree-like approximation.

Although the Tetra-PEG gel is formed by the AB-type coupling of tetra-arm polymers, we can treat this coupling as AA-type coupling of tetra-arm polymers when we consider the stoichiometric conditions. ${ }^{14}$ As for AA-type coupling of tetra-arm polymers, the probability that an arm does not lead to an infinite network $\left(P_{\infty}\right)$ is correlated with $p$ by ${ }^{23}$

$$
P_{\infty}=p \cdot P_{\infty}^{3}+(1-p)
$$

In terms of $P_{\infty}$, the probability that a given tetra-arm polymer has a degree of connection of $n\left(P\left(X_{n}\right)\right)$ is given by

$$
P\left(X_{n}\right)={ }_{4} \mathrm{C}_{n} P_{\infty}^{(4-n)}\left\{1-P_{\infty}\right\}^{n}
$$

where ${ }_{x} \mathrm{C}_{y}$ is the usual notation for the number of combinations of $x$ items taken $y$ at a time: $x ! / y$ ! $(x-y)$ !. Here, it should be noted that only if $n \geqslant 3$ the connections do become cross-links. From Equation 4, the probabilities of a tri-functional crosslink $\left(P\left(X_{3}\right)\right)$ and a tetrafunctional crosslink $\left(P\left(X_{4}\right)\right)$ are calculated as follows:

$$
\begin{aligned}
& P\left(X_{3}\right)={ }_{4} \mathrm{C}_{3} P_{\infty}\left\{1-P_{\infty}\right\}^{3} \\
& P\left(X_{4}\right)=\left\{1-P_{\infty}\right\}^{4}
\end{aligned}
$$

With Equations 5 and $6, \mu$ and $v$ are predicted as follows:

$$
\begin{aligned}
& \mu=\rho\left\{P\left(X_{3}\right)+P\left(X_{4}\right)\right\} \\
& \nu=\rho\left\{\frac{3}{2} P\left(X_{3}\right)+\frac{4}{2} P\left(X_{4}\right)\right\}
\end{aligned}
$$

where $\rho$ is the number density of the Tetra-PEG prepolymers.

The variations of $G, G_{\mathrm{af}}$ and $G_{\mathrm{ph}}$ as a function of $\phi_{0}$ in the $5-10 \mathrm{k}$, $5-20 \mathrm{k}$ and $10-20 \mathrm{k}$ Tetra-PEG gels are shown in Figure 3. $G_{\mathrm{af}}$ and $G_{\mathrm{ph}}$ increased linearly as $\phi_{0}$ increased; this increase reflects the constant $p$. In the $5-20 \mathrm{k}$ Tetra-PEG gels (Figure $3 \mathrm{~b}$ ), $G$ and $G_{\mathrm{ph}}$ agreed with each other throughout the range; this agreement suggests
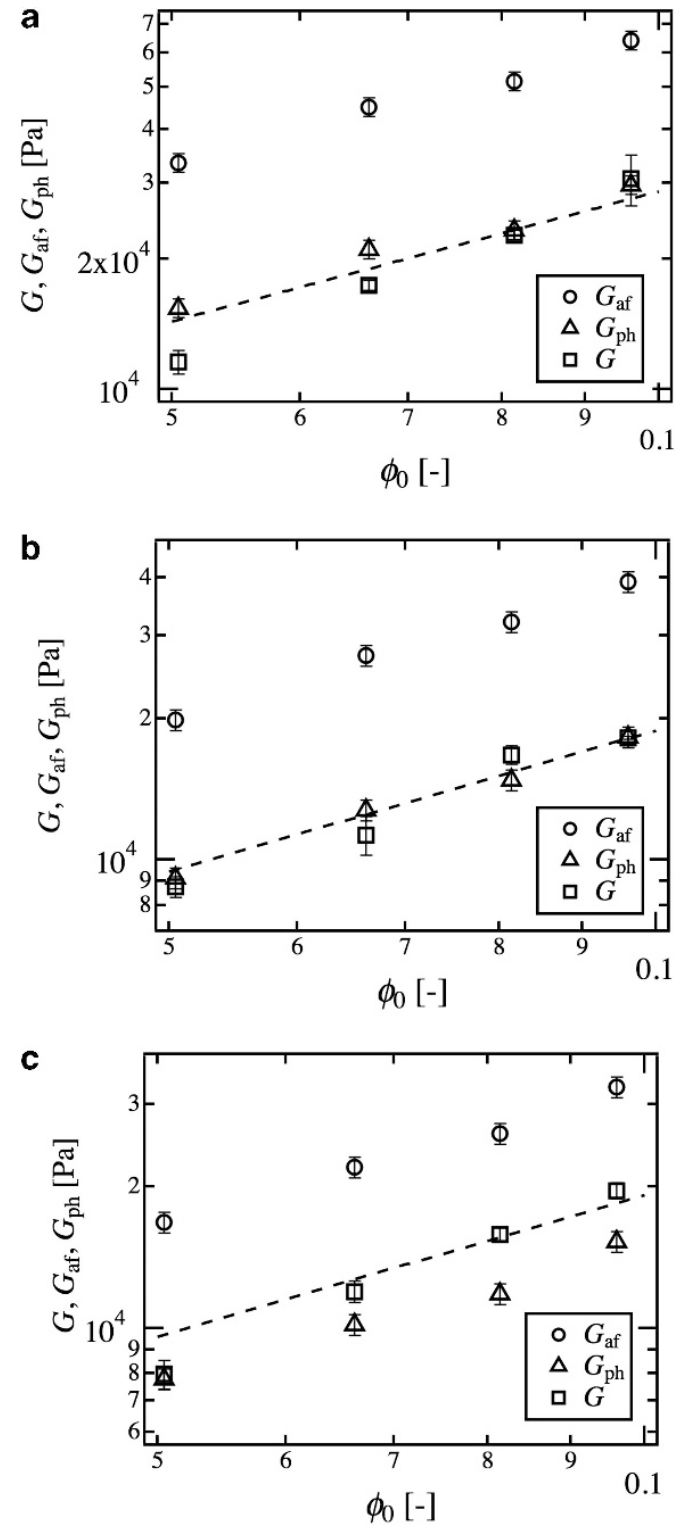

Figure 3 The value of $G$ (squares) estimated from the stretching measurements, $G_{\mathrm{af}}$ (circles) and $G_{\mathrm{ph}}$ (triangles), estimated from the reaction efficiency $(p)$ as a function of $\phi_{0}$ in the (a) $5 k-10 k$, (b) $5 k-20 k$ and (c) $10 k-20 k$ Tetra-PEG gels. The dashed line is the guide showing the relation $G \sim \phi_{0}$.

that the elasticities are roughly predicted by the phantom network model. In the 5-10k Tetra-PEG gels (Figure 3a), the downward deviation of $G$ from $G_{\mathrm{ph}}$ became increasingly pronounced as $\phi_{0}$ decresed; this result suggests that elastically ineffective loops were formed in the low $\phi_{0}$ range. $^{24}$ It seems, however, that $G$ approaches $G_{\mathrm{ph}}$ asymptotically in the high $\phi_{0}$ region, similar to the result for $5 \mathrm{k}$ Tetra-PEG gels. In contrast, in the 10-20 k Tetra-PEG gels (Figure 3c), $G$ was slightly larger than $G_{\mathrm{ph}}$. In our previous paper, we observed the master relation between $G / G_{\text {af }}$ and $\phi_{0} / \phi^{*}$, where $\phi^{*}$ is the overlapping concentration of prepolymers. The shift from the phantom to affine network models was clearly observed from the plot of this relation. ${ }^{25}$ Thus, we performed the same plot for the $5-10 \mathrm{k}, 5-20 \mathrm{k}$ and $10-20 \mathrm{k}$ Tetra-PEG gels (Figure 4). Here, we calculated $\phi^{*}$ for the 


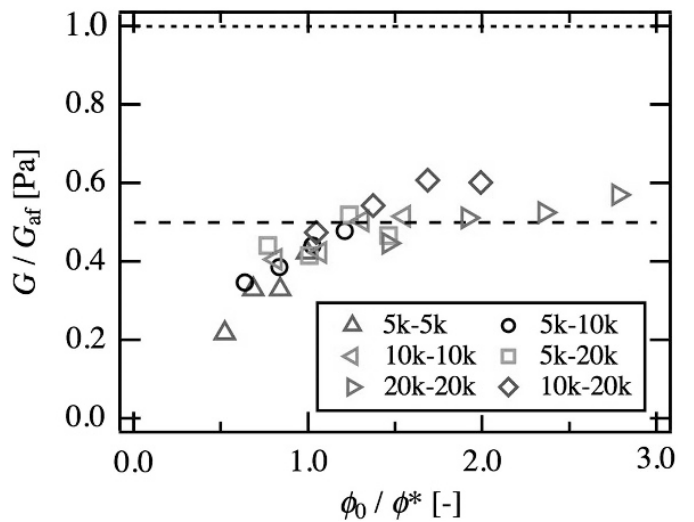

Figure 4 The value of $G / G_{\text {af }}$ as a function of $\phi_{0} / \phi^{*}$ for the Tetra-PEG gels $(5 \mathrm{k}-5 \mathrm{k}$, triangles; $10 \mathrm{k}-10 \mathrm{k}$, left triangles; $20 \mathrm{k}-20 \mathrm{k}$, right triangles; $5 \mathrm{k}-$ $10 k$, circles; $5 k-20 k$, squares; $10 k-20 k$, rhombuses). The data for the $5 k-5 k, 10 k-10 k$ and $20 k-20 k$ Tetra-PEG gels were taken from the literature. ${ }^{25}$ The dashed and dotted lines are the guides showing G/ $G_{\text {af }}=0.5$ and 1.0, respectively. The experimental errors were smaller than the sizes of the symbols. A full color version of this figure is available at Polymer Journal online.

mixed solutions of the $5 \mathrm{k}, 10 \mathrm{k}$ and $20 \mathrm{k}$ Tetra-PEG prepolymers from the formula:

$$
\phi^{*}=\left(\phi_{\mathrm{A}}^{*}+\phi_{\mathrm{B}}^{*}\right) / 2
$$

where $\phi^{*}$ and $\phi_{B}^{*}$ are the overlapping concentrations of the prepolymers $\mathrm{A}$ and $\mathrm{B}$, respectively (Table 1 ). In this plot, the phantom and affine network model predictions are the horizontal lines at $G$ / $G_{\mathrm{af}}=0.5$ (dashed line) and 1 (dotted line), respectively. The results for the $5 \mathrm{k}, 10 \mathrm{k}$ and $20 \mathrm{k}$ Tetra-PEG gels are reproduced from our previous paper. ${ }^{25}$ The results of the size-mismatched Tetra-PEG gels, especially the 5-10 k and 5-20k Tetra-PEG gels, fall onto a master relation; this result suggests that the elasticities of these gels can be predicted using the phantom network model. This result corresponds well to Flory's prediction that phantom-like behavior appears in the fairy dilute regime. ${ }^{26}$ The deviation below $\phi^{*}$ is responsible for the formation of elastically ineffective loops, as discussed in our previous paper. However, in the $10-20 \mathrm{k}$ Tetra-PEG gels, $G / G_{\text {af }}$ is slightly higher than the master relation. Although the discrepancy is not large, there is a possibility that the mixing of size-mismatched prepolymers affects the elasticity. To investigate this point in detail, we investigated the fracture energy and the ultimate elongation.

\section{Fracture energy}

We performed the tearing measurements for the 5-10k, 5-20k and $10-20 \mathrm{k}$ Tetra-PEG gels to investigate the intrinsic fracture energy $\left(T_{0}\right)$. The fracture energy is defined as the energy required for the development of a crack with a unit length. ${ }^{27,28}$ The Lake-Thomas model is a popular model for the prediction of the fracture energy of elastomers and well predicted the fracture energy of conventional Tetra-PEG gels. ${ }^{25}$ In the Lake-Thomas model, ${ }^{29} T_{0}$ is estimated to be the energy needed to break the chemical bonds on the fracture surface and is given by

$$
T_{0}=N U \cdot d \cdot v
$$

where $N$ is the degree of polymerization between neighboring crosslinks, $U$ is the energy required to rupture a monomer unit and $d$ is the displacement length. In our previous treatment, we assumed the absence of trapped entanglement: $v \sim \phi_{0} / N$ and $d \sim N^{1 / 2}$. Hence,

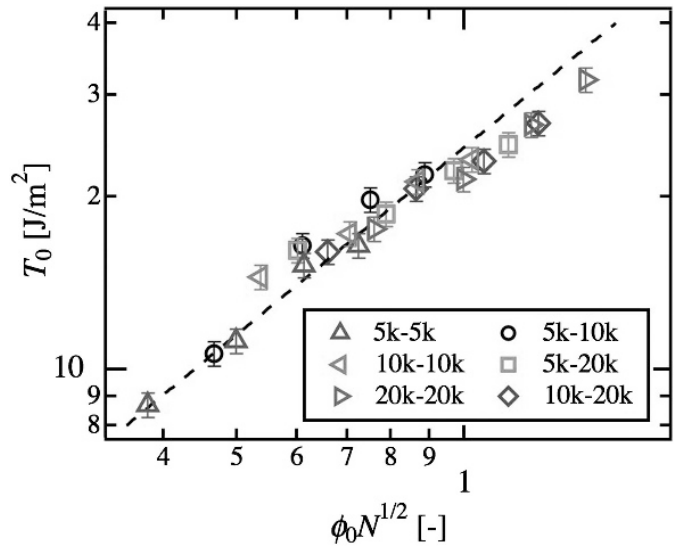

Figure 5 Fracture energy as a function of $\phi_{0} N^{1 / 2}$ for the Tetra-PEG gels $(5 \mathrm{k}-5 \mathrm{k}$, triangles; $10 \mathrm{k}-10 \mathrm{k}$, left triangles; $20 \mathrm{k}-20 \mathrm{k}$, right triangles; $5 \mathrm{k}-10 \mathrm{k}$, circles; $5 \mathrm{k}-20 \mathrm{k}$, squares; $10 \mathrm{k}-20 \mathrm{k}$, rhombuses). The data for the $5 k-5 k, 10 k-10 k$ and $20 k-20 k$ Tetra-PEG gels were taken from the literature. ${ }^{25}$ The dashed lines are the guides showing $T_{0} \sim \phi_{0} N^{1 / 2}$. A full color version of this figure is available at Polymer Journal online.

$T_{0}$ scales with the $\phi_{0}$ and $N$ by

$$
T_{0} \sim \phi_{0} N^{1 / 2}
$$

The values of $T_{0}$ for the $5-10 \mathrm{k}, 5-20 \mathrm{k}$ and $10-20 \mathrm{k}$ Tetra-PEG gels are shown as a function of $\phi_{0} N^{1 / 2}$ in Figure 5. The data for the $5-5 \mathrm{k}$, $10-10 \mathrm{k}$ and $20-20 \mathrm{k}$ Tetra-PEG gels are taken from our previous report. ${ }^{25}$ The data for size-mismatched Tetra-PEG gels fell onto the master curve showing $T_{0} \sim \phi_{0} N^{1 / 2}$; this result suggests the absence of trapped entanglements and the applicability of the Lake-Thomas model, similar to conventional Tetra-PEG gels. Although there is an ambiguity because of experimental error, these data indicate that all the samples, including the $10-20 \mathrm{k}$ Tetra-PEG gel, have almost no trapped entanglement.

\section{Ultimate elongation ratio}

Finally, we investigated the ultimate elongation ratio. The ultimate elongation ratio is estimated from the stress-elongation relation with the extended Gent model, whose applicability was confirmed in our previous studies. ${ }^{30}$ The extended Gent model predicts uniaxial stretching behaviors as follows: ${ }^{31}$

$$
\begin{aligned}
& \sigma=\frac{\alpha \cdot G\left(\lambda-\lambda^{-2}\right)}{1-\left(\lambda^{2}-2 \lambda^{-1}-3\right) / l_{m}}+(1-\alpha) \cdot G\left(1-\lambda^{-3}\right) \\
& l_{\mathrm{m}}=\left(\lambda_{\text {max }}^{2}+\lambda_{\text {max }}^{-1}\right)-3
\end{aligned}
$$

where $\lambda_{\max }$ is the ultimate elongation, and the parameter $\alpha$ was estimated from the fit to the stress-elongation curves for the Tetra-PEG gels $(20 \mathrm{k})$ under biaxial stretching. Because $G$ is fixed to be the value obtained from the linear fit, the only fitting parameter is $\lambda_{\text {max }}$.

The fit results for the $5-10 \mathrm{k}, 5-20 \mathrm{k}$ and $10-20 \mathrm{k}$ Tetra-PEG gels are shown in Figure 6. Regardless of $\phi_{0}$ and prepolymer combinations, the stress-elongation relations are well predicted by the extended Gent model; this result suggests that the uniaxial stretching behaviors of the size-mismatched Tetra-PEG gels are similar to those of the conventional Tetra-PEG gels.

The values of $\lambda_{\max }$ for the $5-10 \mathrm{k}$ and $10-20 \mathrm{k}$ Tetra-PEG gels are shown as a function of $\phi_{0}$ in Figures $7 \mathrm{a}$ and $\mathrm{b}$, respectively. 

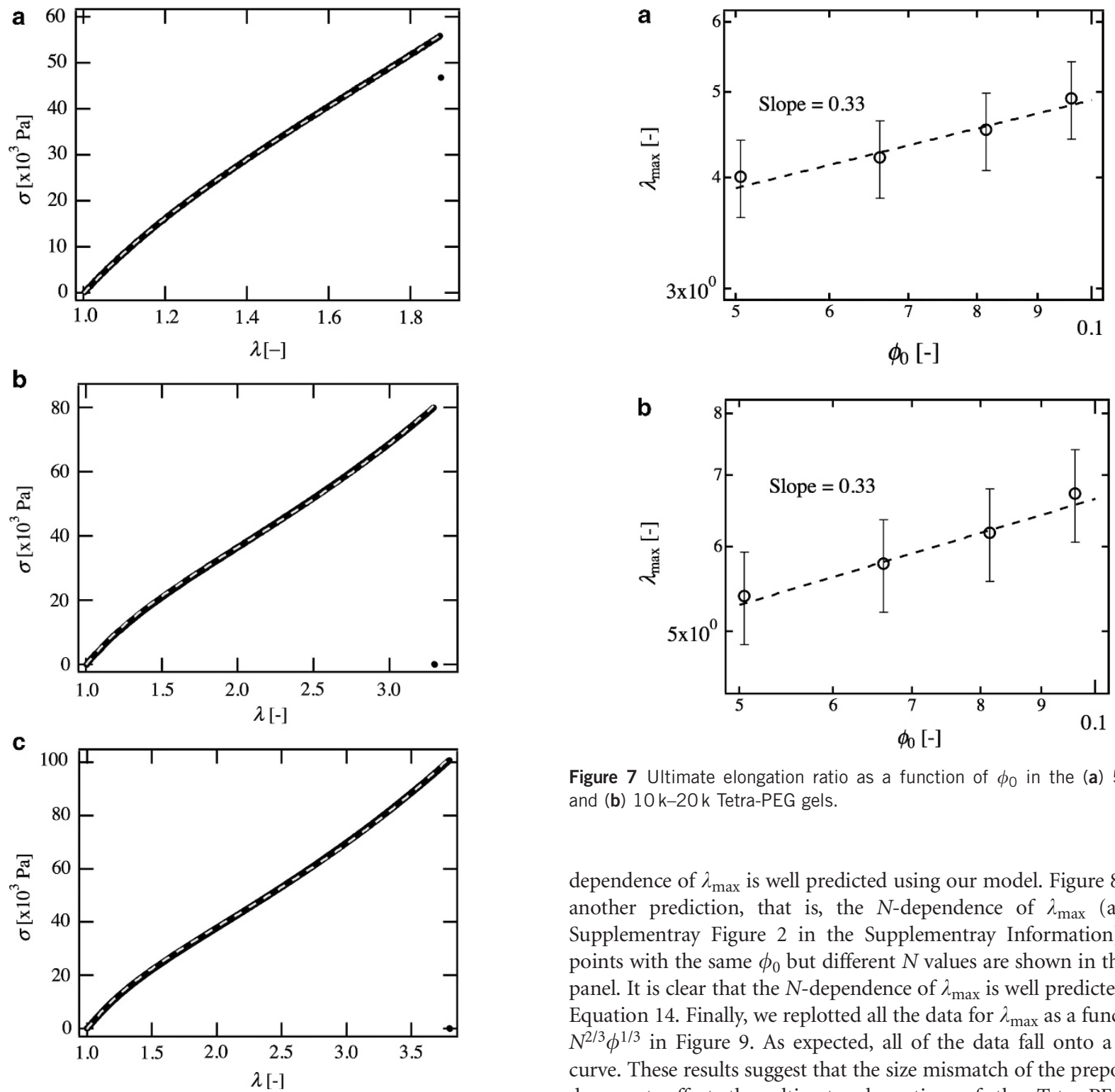

Figure 6 Stress-elongation curves in the (a) $5 k-10 k$, (b) $5 k-20 k$ and (c) $10 \mathrm{k}-20 \mathrm{k}$ Tetra-PEG gels $\left(\phi_{0}=0.096\right)$.

$\lambda_{\max }$ increased as $\phi_{0}$ increased (also see Supplementray Figure 1 in the Supplementray Information). These results differ from the prediction of the Kuhn model, which predicts $\lambda_{\max } \sim N^{1 / 2}$ and 'no' dependence on $\phi_{0},{ }^{32}$ and they correspond to our results for conventional TetraPEG gels. ${ }^{30}$

In our previous study, we proposed a novel semi-empirical model that predicts the $\lambda_{\max }$ of conventional Tetra-PEG gels: ${ }^{30}$

$$
\lambda_{\max } \sim \frac{L}{l_{g}} \sim \frac{a N}{v^{-1 / 3}} \sim \phi_{0}^{1 / 3} N^{2 / 3}
$$

where $L$ is the contour length, $l_{\mathrm{g}}$ is the geometrical distance between neighboring crosslinks and $a$ is the monomer length. The only difference between the Kuhn model and our model is the denominator, $l_{\mathrm{g}}$, which is calculated as $l_{\mathrm{g}} \sim v^{-1 / 3}$.

The prediction from Equation 14 is shown as a guide in Figures $7 \mathrm{a}$ and $\mathrm{b}$ and corresponds well to the experimental results; the $\phi_{0^{-}}$

Figure 7 Ultimate elongation ratio as a function of $\phi_{0}$ in the (a) $5 k-10 k$ and (b) $10 \mathrm{k}-20 \mathrm{k}$ Tetra-PEG gels.

dependence of $\lambda_{\max }$ is well predicted using our model. Figure 8 shows another prediction, that is, the $N$-dependence of $\lambda_{\max }$ (also see Supplementray Figure 2 in the Supplementray Information). Data points with the same $\phi_{0}$ but different $N$ values are shown in the same panel. It is clear that the $N$-dependence of $\lambda_{\max }$ is well predicted using Equation 14. Finally, we replotted all the data for $\lambda_{\max }$ as a function of $N^{2 / 3} \phi^{1 / 3}$ in Figure 9. As expected, all of the data fall onto a master curve. These results suggest that the size mismatch of the prepolymers does not affect the ultimate elongation of the Tetra-PEG gels. These results also support the validity of our prediction that the ultimate elongation depends on $\phi_{0}$ and $N$, and this dependence is $\lambda_{\text {max }} \sim \phi_{0}{ }^{1 / 3} N^{2 / 3}$. It should be noted that the $10-20 \mathrm{k}$ samples also obeyed the prediction of Equation 14. Considering all the data, we conclude that the size mismatch of the prepolymers does not affect their structure and mechanical properties.

\section{CONCLUSION}

In conclusion, the elastic modulus, the fracture energy and the ultimate elongation ratio of the size-mismatched Tetra-PEG gels have tendencies similar to those of conventional Tetra-PEG gels. From these results, we conclude that the size-mismatched Tetra-PEG gels have the same degree of homogeneity as the conventional Tetra-PEG gels, and the difference in prepolymer size does not affect the homogeneity. It should be noted that the small prepolymer itself induces the formation of elastically ineffective loops, as shown with the $5 \mathrm{k}$ Tetra-PEG gels. The formation of elastically ineffective loops is strongly related to the normalized polymer volume fraction $\left(\phi_{0} / \phi^{*}\right)$. In the range investigated in this study, the effect of elastically ineffective loops is negligible above the overlapping volume fraction $\left(\phi_{0} / \phi^{\star}>1\right)$. Thus, we expected to be 

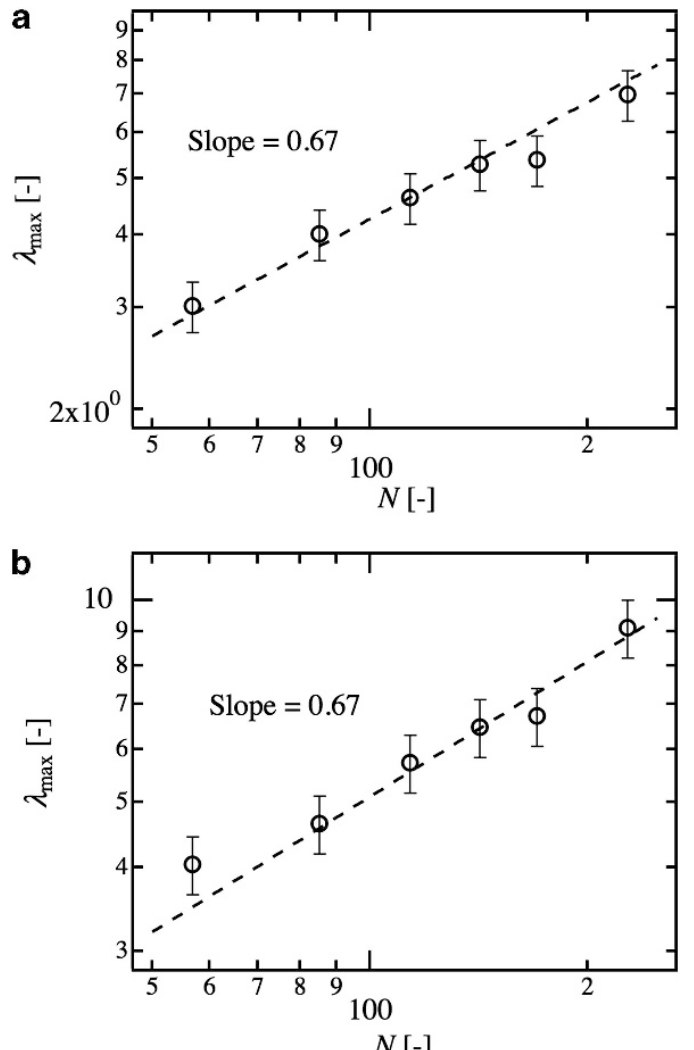

$N[-]$

Figure 8 Ultimate elongation ratio as a function of $N$ in the Tetra-PEG gels $\left(\mathbf{a}, \phi_{0}=0.050\right.$; and $\left.\mathbf{b}, \phi_{0}=0.096\right)$. The data for the $5 \mathrm{k}-5 \mathrm{k}, 10 \mathrm{k}-10 \mathrm{k}$ and $20 \mathrm{k}-20 \mathrm{k}$ Tetra-PEG gels were taken from the literature. ${ }^{30}$

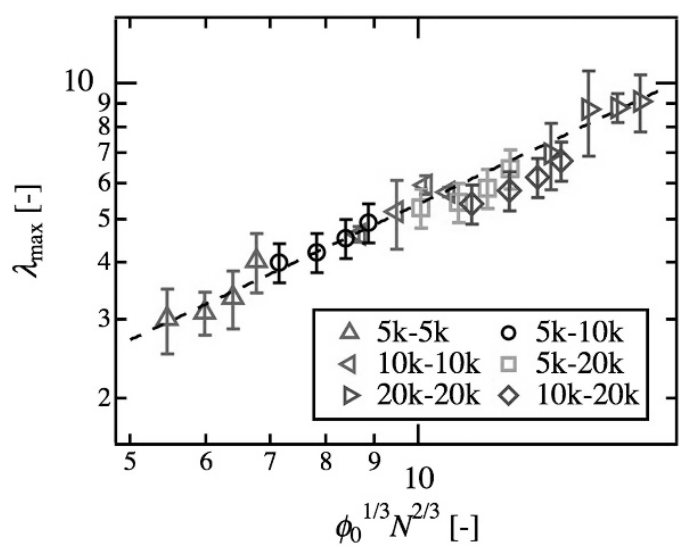

Figure 9 Ultimate elongation ratio as a function of $\phi_{0}{ }^{1 / 3} N^{2 / 3}$ for the TetraPEG gels $(5 k-5 k$, triangles; $10 k-10 k$, left triangles; $20 k-20 k$, right triangles; $5 k-10 k$, circles; $5 k-20 k$, squares; $10 k-20 k$, rhombuses). The data for the $5 k-5 k, 10 k-10 k$ and $20 k-20 k$ Tetra-PEG gels were taken from the literature..$^{30}$ The dashed lines are the guides showing $T_{0} \sim \phi_{0}{ }^{1 / 3}$ $N^{2 / 3}$. A full color version of this figure is available at Polymer Journal online.

able to form homogeneous polymer networks from the prepolymers with the 'same functionalities' and with molecular weights from $5 \mathrm{k}$ to $40 \mathrm{k}$ above the overlapping polymer volume fraction. The effects of the prepolymer functionality will be discussed in our forthcoming paper.

\section{CONFLICT OF INTEREST}

The authors declare no conflict of interest.

\section{ACKNOWLEDGEMENTS}

This work was supported by the Japan Society for the Promotion of Science (JSPS) through Grants-in-Aid for Scientific Research, the Center for Medical System Innovation (CMSI), the Graduate Program for Leaders in Life Innovation (GPLLI), the International Core Research Center for Nanobio and the Funding Program for World-Leading Innovative R\&D on Science and Technology (FIRST program); the Ministry of Education, Culture, Sports, Science and Technology in Japan (MEXT) through the Center for NanoBio Integration (CNBI); the Japan Science and Technology Agency (JST) through the S-innovation program; and Grants-in-Aid for Scientific Research from the Ministry of Education, Culture, Sports, Science and Technology (no. 23700555 to TS and no. 24240069 to UC).

1 Baumann, G. \& Chrambach, A. A highly crosslinked, transparent polyacrylamide gel with improved mechanical stability for use in isoelectric focusing and isotachophoresis. Anal. Biochem. 70, 32-38 (1976).

2 Hochstrasser, D. F., Patchornik, A. \& Merril, C. R. Development of polyacrylamide gels that improve the separation of proteins and their detection by silver staining. Anal. Biochem. 173, 412-423 (1988).

3 Cohen, Y., Ramon, O., Kopelman, I. J. \& Mizrahi, S. Characterization of inhomogeneous polyacrylamide hydrogels. J. Polym. Sci. Part B 30, 1055-1067 (1992).

4 Hsu, T.-P., Ma, D. S. \& Cohen, C. Effects of inhomogeneities in polyacrylamide gels on thermodynamic and transport properties. Polymer. (Guildf). 24, 1273-1278 (1983).

5 Shibayama, M. Spatial inhomogeneity and dynamic fluctuations of polymer gels. Macromol. Chem. Phys. 199, 1-30 (1998).

6 Ikkai, F. \& Shibayama, M. Inhomogeneity control in polymer gels. J. Polym. Sci. Part B 43, 617-628 (2005)

7 Hild, G. Model networks based on 'endlinking' processes: synthesis, structure and properties. Prog. Polym. Sci. 23, 1019-1149 (1998).

8 Shibayama, M., Takahashi, H. \& Nomura, S. Small-angle neutron-scattering study on end-linked poly(tetrahydrofuran) networks. Macromolecules 28, 6860-6864 (1995).

9 Michalke, W., Lang, M., Kreitmeier, S. \& Goritz, D. Comparison of topological properties between end-linked and statistically cross-linked polymer networks. J. Chem. Phys. 117, 6300-6307 (2002).

10 Sakai, T., Matsunaga, T., Yamamoto, Y., Ito, C., Yoshida, R. \& Suzuki, S. et al. Design and fabrication of a high-strength hydrogel with ideally homogeneous network structure from tetrahedron-like macromonomers. Macromolecules 41, 5379-5384 (2008).

11 Sakai, T., Akagi, Y., Matsunaga, T., Kurakazu, M., Chung, U. \& Shibayama, M. Highly Elastic and Deformable Hydrogel Formed from Tetra-arm Polymers. Macromol. Rapid Commun. 31, 1954-1959 (2010).

12 Sakai, T. Gelation mechanism and mechanical properties of Tetra-PEG gel. React. Funct. Polym. 73, 898-903 (2013).

13 Matsunaga, T., Sakai, T., Akagi, Y., Chung, U. \& Shibayama, M. Structure Characterization of Tetra-PEG Gel by Small-Angle Neutron Scattering. Macromolecules 42, 1344-1351 (2009).

14 Akagi, Y., Matsunaga, T., Shibayama, M., Chung, U. \& Sakai, T. Evaluation of Topological Defects in Tetra-PEG Gels. Macromolecules 43, 488-493 (2010).

15 Akagi, Y., Katashima, T., Katsumoto, Y., Fujii, K., Matsunaga, T. \& Chung, U. et al. Examination of the Theories of Rubber Elasticity Using an Ideal Polymer Network. Macromolecules 44, 5817-5821 (2011).

16 Matsunaga, T., Sakai, T., Akagi, Y., Chung, U. I. \& Shibayama, M. SANS and SLS Studies on Tetra-Arm PEG Gels in As-Prepared and Swollen States. Macromolecules 42, 6245-6252 (2009).

17 Matsunaga, T., Asai, H., Akagi, Y., Sakai, T., Chung, U. \& Shibayama, M. SANS Studies on Tetra-PEG Gel under Uniaxial Deformation. Macromolecules 44, 1203-1210 (2011).

18 Lange, F., Schwenke, K., Kurakazu, M., Akagi, Y., Chung, U. I. \& Lane, M. et al. Connectivity and Structural Defects in Model Hydrogels: A Combined Proton NMR and Monte Carlo Simulation Study. Macromolecules 44, 9666-9674 (2011).

19 Nishi, K., Chijiishi, M., Katsumoto, Y., Nakao, T., Fujii, K. \& Chung, U. et al. Rubber elasticity for incomplete polymer networks. J. Chem. Phys. 137 (2012).

20 Kurakazu, M., Katashima, T., Chijiishi, M., Nishi, K., Akagi, Y. \& Matsunaga, T. et al. Evaluation of Gelation Kinetics of Tetra-PEG Gel. Macromolecules 43, 3935-3940 (2010).

21 Nishi, K., Fujii, K., Chijiishi, M., Katsumoto, Y., Chung, U. \& Sakai, T. et al. Kinetic Study for AB-Type Coupling Reaction of Tetra-Arm Polymers. Macromolecules 45, 1031-1036 (2012).

22 Landau, L. D., Lifshits, E. M., Kosevich, A. M. \& Pitaevskii, L. P. Theory of elasticity (Pergamon Press, Oxford, UK, and New York, 1986).

23 Miller, D. R. \& Macosko, C. W. New Derivation of Post Gel Properties of Network Polymers. Macromolecules 9, 206-211 (1976).

24 Vasiliev, V. G., Rogovina, L. Z. \& Slonimsky, G. L. Dependence of Properties of Swollen and Dry Polymer Networks on the Conditions of Their Formation in Solution. Polymer. (Guildf). 26, 1667-1676 (1985).

25 Akagi, Y., Gong, J. P., Chung, U. \& Sakai, T. Transition between phantom and affine network model observed in polymer gels with controlled network structure. Macromolecules 46, 1035-1040 (2013). 
26 Flory, P. J. Theory of elasticity of polymer networks - effect of local constraints on junctions. J. Chem. Phys. 66, 5720-5729 (1977).

27 deGennes, P. G. Soft adhesives. Langmuir. 12, 4497-4500 (1996).

28 Tanaka, Y., Kuwabara, R., Na, Y. H., Kurokawa, T., Gong, J. P. \& Osada, Y. Determination of fracture energy of high strength double network hydrogels. J. Phys. Chem. B 109 (2005)

29 Lake, G. J. \& Thomas, A. G. Strength of Highly Elastic Materials. Proc. R. Soc. A 300, 108-10 (1967).
30 Akagi, Y., Katashima, T., Sakurai, H., Chung, U. \& Sakai, T. Ultimate elongation of polymer gels with controlled network structure. RSC Advances 3, 13251-13258 (2013).

31 Katashima, T., Urayama, K., Chung, U. I. \& Sakai, T. Strain energy density function of a near-ideal polymer network estimated by biaxial deformation of Tetra-PEG gel. Soft Matter 8, 8217-8222 (2012).

32 Kuhn, W. Dependence of the average transversal on the longitudinal dimensions of statistical coils formed by chain molecules. J. Polym. Sci. 1, 380-388 (1946).

Supplementary Information accompanies the paper on Polymer Journal website (http://www.nature.com/pj) 